\title{
Study of the synthesis of novel trisubstituted acridines
}

\author{
Maria Matejova, Ladislav Janovec,* and Jan Imrich \\ P. J. Safarik University, Faculty of Science, Department of Organic chemistry, Moyzesova 11, \\ 04001 Kosice, Slovak Republic \\ E-mail: ladislav.janovec@upjs.sk
}

DOI:http://dx.doi.org/10.3998/ark.5550190.p009.033

\begin{abstract}
An efficient methodology has been developed for the synthesis of 3,6-diamino-9[(phenylalkyl)amino]acridines by heating 2-propanol solutions of 3,6-di(butanoylamino)-9[(phenylalkyl)amino]acridines in the presence of $\mathrm{NaOH}$. 3,6-Diamino-9[(phenylalkyl)amino]acridines were used in the synthesis of final products 3,6-bis\{3-[2(dimethylamino)ethyl]ureido\}-9-[(phenylalkyl)amino]acridines. Herein we rationalize the formation of 3,6,9-triaminoacridine and propose the reaction mechanism for the observed transformation.
\end{abstract}

Keyword: Acridine, acridone, thiourea, urea

\section{Introduction}

Acridine derivatives have been extensively studied for their chemotherapeutic properties due to their ability to intercalate into DNA and disrupt unwanted cellular processes. ${ }^{1,2}$ In a more recent study, it has been reported that quadruplex-binding trisubstituted acridines have exhibited rapid anti-tumor effects involving several parallel mechanisms, including telomere uncapping, direct or indirect telomerase inhibition with the characteristic induction of senescence and apoptosis. ${ }^{3-8}$ Recent research has also shown that G-quadruplexes selectively target both telomerase and telomere in cancer cells. ${ }^{9-11}$ Beyond these classical applications as drugs and as ligands, acridine derivatives have also been used as fluorescent probes and chemosensors. ${ }^{12}$

Taking this into consideration, structural modifications of acridine scaffold became widely studied by organic chemists. An extensive work in this field led to new synthetic inventions enabling the preparation of acridine skeleton with a broad structural diversity. Here, we would like to present results related to an attempt to prepare a new series of trisubstituted acridines based on BRACO-19 framework as G-quadruplex targeting compounds (Figure 1). Structural similarities between BRACO-19 framework and our new structures are based on the 3,6,9- 
derivatisation of acridine scaffold. Whereas positions 3,6- are modified by aminoaliphatic groups, the position 9 - is substituted by phenylalkylamino moiety to provide better flexibility.
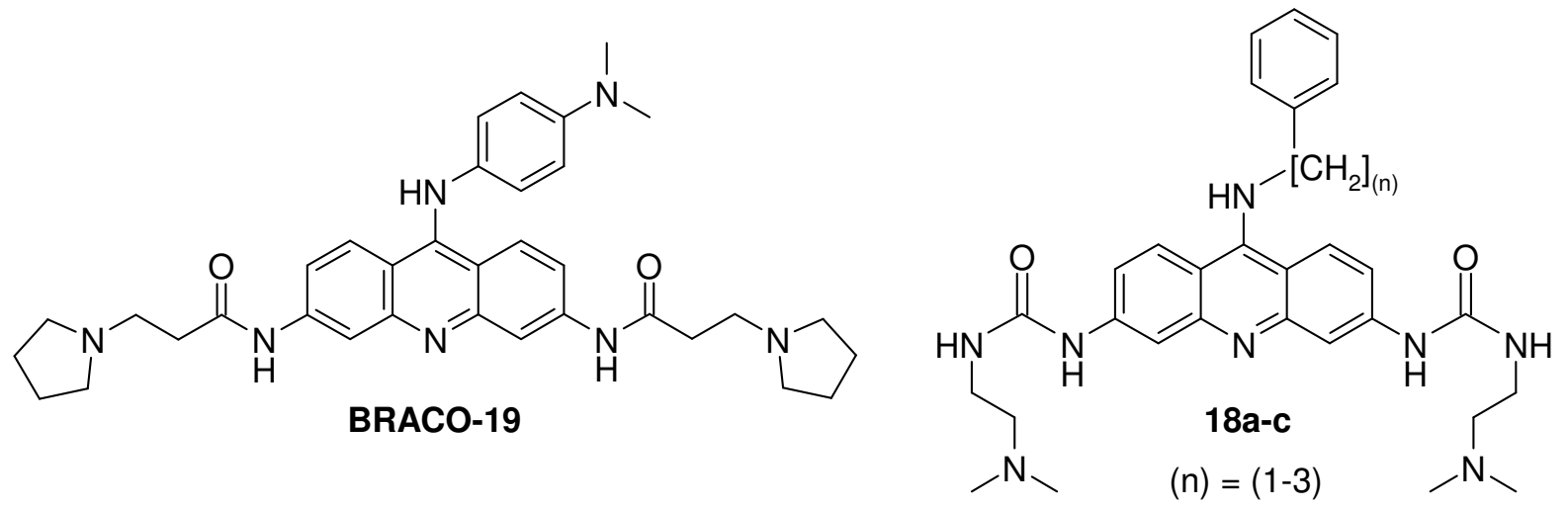

Figure 1. BRACO-19 and novel trisubstituted acridines 18a-c.

\section{Results and Discussion}

\section{Preparation of acridone}

A synthetic pathway described herein starts by the preparation of 3,6-diaminoacridone 4 and provides access to the trisubstituted acridine framework. Preparation of 3,6-diaminoacridone 4 was done in accordance to previously described procedure based on diphenylmethane (1) (Scheme 1). ${ }^{13}$ The overall synthetic protocol was adjusted to simplify each reaction step and to enhance the overall yield. The initial step, nitration of diphenylmethane (1), proceeded in a yield similar to published results. The subsequent oxidation of $\mathbf{2}$ was modified due to poor solubility of reactant in acetic acid as stated in the original report. The amount of acetic acid had to be increased at least three times. Subsequent cyclization of compound $\mathbf{3}$ was improved substantially. Shortly, the amount of hydrochloric acid and ethanol was reduced, anhydrous stannous chloride was replaced by the cheaper dihydrate, and the reaction mixture was continuously refluxed for 18 hours. With these modifications, previously reported yield increased from $60 \%$ to $80 \%$. Modified synthetic methodology provided $20 \%$ increase of the overall yield.

A key intermediate $\mathbf{6}$ was synthesized by the reaction of $\mathrm{POCl}_{3}$ with precursor 5 which was in turn obtained by the reaction of acridone 4 with butyric anhydride at elevated temperature. Installation of the butanoyl protective group was performed with the aim to improve the solubility of the intermediate $\mathbf{6}$. Resulting derivative $\mathbf{6}$ was then purified by crystallization from ethyl acetate. The synthesis of trisubstituted compounds 7a-c involved treatment of 6 with phenylalkyl amines (Scheme 2). Next, there was intended to remove the protecting groups from derivates 7a-c with the intention to obtain amines. There was considered the use of acidic conditions to obtain the free amine 8a. Interestingly, this reaction afforded 3,6,9-triaminoacridine 
(9) and desired product 8a was neither detected nor isolated (Scheme 3). This unexpected result prompted us to elucidate the reaction conditions that could lead to the formation of derivative 8a. Interestingly, neither a change of the concentration of sulfuric acid nor its replacement by other mineral acid influenced the outcome of the reaction.<smiles>CC(C)(C)CC(C)(C)COc1ccc(Cc2ccc([N+](=O)[O-])cc2[N+](=O)[O-])c([N+](=O)[O-])c1</smiles>

i. $\mathrm{H}_{2} \mathrm{SO}_{4}, \mathrm{KNO}_{3}, 70^{\circ} \mathrm{C}, 40$ min., $84 \%$;

ii. $\mathrm{CrO}_{3}, \mathrm{CH}_{3} \mathrm{CO}_{2} \mathrm{H}$, reflux, $3 \mathrm{hr}$., $94 \%$;

iii. $\mathrm{SnCl}_{2} \times 2 \mathrm{H}_{2} \mathrm{O}, \mathrm{HCl}, \mathrm{EtOH}, 90{ }^{\circ} \mathrm{C}, 18 \mathrm{hr}$., $80 \%$.<smiles></smiles>

Scheme 1. Synthesis of 3,6-diamino-9(10H)acridone (4).<smiles>Nc1ccc2c(=O)c3ccc(N)cc3[nH]c2c1</smiles>

4<smiles>CCCC(=O)Nc1ccc2c(NC)c3ccc(NC(=O)C(C)C)cc3nc2c1</smiles>
7a-c<smiles>CCCCC(=O)Nc1ccc2c(=O)c3ccc(NC(=O)CCC)cc3[nH]c2c1</smiles>
5 ii. ii.<smiles>C#CCPC(=O)Nc1ccc2c(Cl)c3ccc(NC(=O)C(C)C)cc3nc2c1</smiles>

i. butyric anhydride, $100^{\circ} \mathrm{C}, 3 \mathrm{hr}$., $49 \% ; \quad(\mathrm{n})=(1-3)$

ii. $\mathrm{POCl}_{3}, 70{ }^{\circ} \mathrm{C}, 1.5 \mathrm{hr}$., $47 \%$;

iii. $\mathrm{Ph}-\left[\mathrm{CH}_{2}\right]_{(\mathrm{n})}-\mathrm{NH}_{2}$; acetonitrile, reflux, $1 \mathrm{hr}$.

Scheme 2. Synthesis of the trisubstituted acridine derivatives. 


\section{Basic hydrolysis}

Overall, this approach proved to be unsuccessful to produce the desired product 8a despite extensive experimentation. To circumvent this issue, alkaline conditions was used instead, a mixture of aliphatic alcohols with sodium hydroxide. The superior level of conversion was obtained in the case of 2-propanol (Scheme 4, Table 1, entry 5), the use of other alcohols (Table 1) resulted only to partial formation of derivate 8a. The worst result was achieved when a mixture of 2-propanol/water (50\% each) was used as a solvent leading to a complex mixture containing the substrate 7a along with the desired free amine 8a and acridones $\mathbf{4}$ and $\mathbf{5}$ (Scheme 4). To understand this reaction better, 9-(benzylamino)acridine (10) was used as the model compound in order to prevent any possible side reactions.

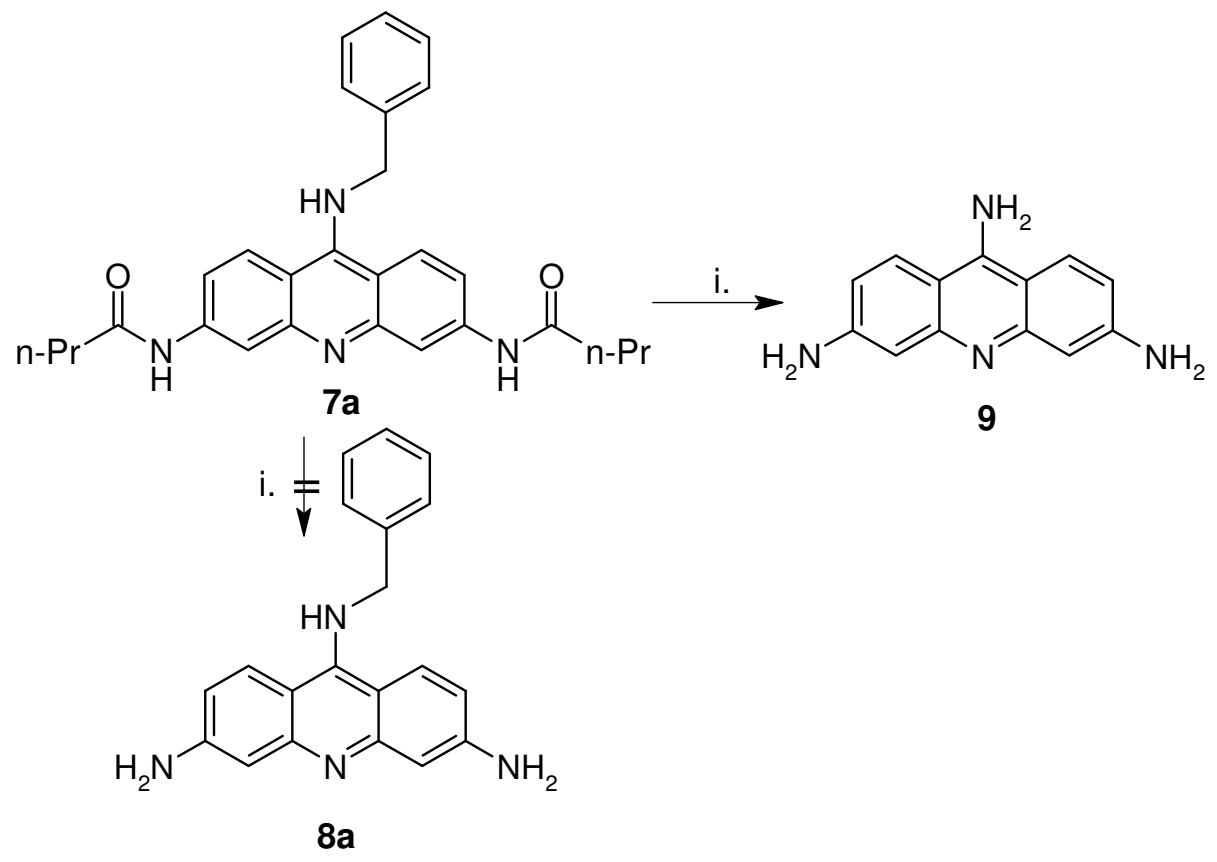

i. $70 \% \mathrm{H}_{2} \mathrm{SO}_{4}, 3 \mathrm{hr} ., 80{ }^{\circ} \mathrm{C}, 80 \%$.

Scheme 3. Products of hydrolysis of acridine derivative 7a.

The derivative $\mathbf{1 0}$ was subjected to a mixture of ethanol/ $\mathrm{NaOH}$ or 2 -propanol $/ \mathrm{NaOH}$ under the same reaction conditions. In the case of ethanol the reaction produced the acridone 10a predominately (Scheme 5). On the other hand, the use of 2-propanol resulted in minimal conversion to acridone 10a (Scheme 5). Observed outcome of the reaction with model substrate might be attributed to different kinetics of the reaction with more sterically hindered 2propanolate versus less crowded ethanolate. This observation might also provide rational for the reaction shown in the Scheme 4. In a mixture of 2-propanol/water, bulky 2-propanolate is formed that attacks predominately 3,6-positions of the acridine ring while avoiding the position 9, whereas rather small hydroxyl ion reacts with no chemoselectivity. 
Table 1. Alkaline hydrolysis of acridine derivative 7a

\begin{tabular}{lccc}
\hline Reaction conditions & & & \\
\hline \multicolumn{1}{c}{ Alcohol } & $\begin{array}{c}\text { Temperature } \\
{\left[{ }^{\circ} \mathrm{C}\right]}\end{array}$ & $\begin{array}{c}\text { Time } \\
{[\text { hours }]}\end{array}$ & Result of hydrolysis \\
2-Propanol $^{\mathrm{a}}$ & 80 & 24 & $\mathbf{7 a}+(\mathbf{8 a}<10 \%)^{\mathrm{d}}+(\mathbf{5}<10 \%)^{\mathrm{d}}+(\mathbf{4}<10 \%)^{\mathrm{d}}$ \\
Methanol $^{\mathrm{b}}$ & reflux & 5 & $\mathbf{7 a}+(\mathbf{8 a}<10 \%)^{\mathrm{d}}$ \\
Ethanol $^{\mathrm{b}}$ & reflux & 5 & $\mathbf{7 a}+(\mathbf{8 a}<20 \%)^{\mathrm{d}}$ \\
1-Propanol $^{\mathrm{b}}$ & 80 & 5 & $\mathbf{7 a}+(\mathbf{8 a}<35 \%)^{\mathrm{d}}$ \\
2-Propanol $^{\mathrm{b}}$ & 80 & 5 & $\mathbf{8 a}>95 \%{ }^{\mathrm{d}}$ \\
Methanol $^{\mathrm{c}}$ & reflux & 5 & $\mathbf{1 5}$ \\
\hline
\end{tabular}

a $1.5 \mathrm{~mL}$ of alcohol, $1.5 \mathrm{~mL}$ of water, $0.03 \mathrm{~mL}$ of concentrated aqueous solution of $\mathrm{NaOH}, 0.1 \mathrm{~g}$ of derivative $\mathbf{7 a}$.

b $3 \mathrm{~mL}$ of alcohol, $0.03 \mathrm{~mL}$ of concentrated aqueous solution of $\mathrm{NaOH}, 0.1 \mathrm{~g}$ of derivative $7 \mathbf{a}$.

c $3 \mathrm{~mL}$ of alcohol, $1 \mathrm{~mL}$ of $25 \% \mathrm{NH}_{4} \mathrm{OH}, 0.1 \mathrm{~g}$ of derivative $7 \mathbf{a}$.

${ }^{\mathrm{d}}$ an approximate ratio obtained from ${ }^{1} \mathrm{H}$ NMR measurements.<smiles></smiles>

i: $2-\mathrm{PrOH} / \mathrm{HOH}(1: 1), \mathrm{NaOH}, 80^{\circ} \mathrm{C}, 24$ hr.; ii: $2-\mathrm{PrOH}, \mathrm{NaOH}, 80^{\circ} \mathrm{C}, 5 \mathrm{hr}$..

Scheme 4. Basic hydrolysis of derivative 7a. 


\section{Acidic hydrolysis}

To find an explanation for the formation of derivative 9 under acidic conditions (Scheme 3), there was made an attempt to elucidate the reaction mechanism. There is outlined two possible reaction pathways which might explain the loss of the benzyl group (Scheme 6.). The first one suggests the formation of benzyl alcohol (12) as the consequence of nuclephilic displacement by water molecule (tautomeric form $\mathbf{B}$, Scheme 6). The second proposal is based on the formation of benzaldehyde (13), the expected product of the hydrolysis of Schiff base-like tautomer $\mathbf{C}$ (Scheme 6). To distinguish between these two possibilities, there was made an attempt to isolate the products of acidic hydrolysis of derivative 10. There were used $50 \%$ aqueous $\mathrm{H}_{2} \mathrm{SO}_{4}, 50 \%$ aqueous $\mathrm{H}_{3} \mathrm{PO}_{4}$ and $35 \%$ aqueous $\mathrm{HCl}$. Substrate 10 was heated for 3 hours at $80{ }^{\circ} \mathrm{C}$, followed by cooling of the mixture, and extraction by diethylether. The residues obtained upon removal of diethylether were analyzed by NMR. Benzyl alcohol (12) was found to be the product of the reaction in all cases as confirmed by NMR (Scheme 6). Benzoic acid (14) was found as the minor product in the case of sulfuric acid likely formed as a product of the oxidation of benzaldehyde (13), that might be produced by hydrolysis of tautomer $\mathbf{C}$ (Scheme 6.).

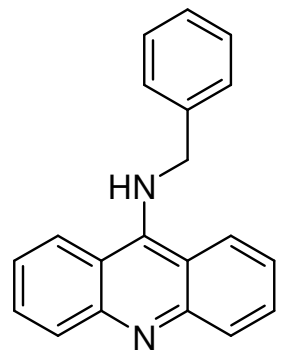

10

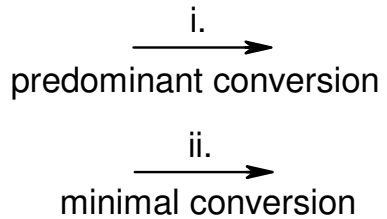

minimal conversion

i. EtOH, $\mathrm{NaOH}, 70{ }^{\circ} \mathrm{C}, 24 \mathrm{hr}$.

ii. 2- $\mathrm{PrOH}, \mathrm{NaOH}, 70^{\circ} \mathrm{C}, 24 \mathrm{hr}$.<smiles>O=c1c2ccccc2[nH]c2ccccc12</smiles>

$10 \mathrm{a}$

Scheme 5. Hydrolysis of compound $\mathbf{1 0 .}$

The rational for the loss of the benzyl group is outlined in Scheme 7. Under acidic conditions, nucleophilic displacement (water as a nucleophile) takes place with 9-aminoacridine (11) as the leaving group. Protonation makes of 9-aminoacridine (11) better leaving group, and the reaction likely occures by the mixed or monomolecular type of mechanism. It is important to stress that above discussed reaction mechanism is drawn with the delocalisation of a partial charge on the phenyl ring, generated during nuclephilic attack on methylene carbon (Scheme 7). This assumption is supported by the fact that derivatives $\mathbf{7 b}$ and $\mathbf{7 c}$ possessing one or two carbon atoms longer side chain do not form triaminoacridine 9 under acidic conditions (Scheme 8). 


\section{Mechanistic studies}

Although there was not detected the presence of derivative $\mathbf{9}$ as one of products of the reaction described in the Scheme 4, this alternative reaction pathway, nucleophilic displacement of acridine framework by a hydroxyl/2-propanolate ion, could not be excluded completely. Computational chemistry calculation was performed to understand the reaction in more details. There was intended to study the bimolecular nucleophilic displacement on the methylene carbon of the model compound 9-(benzylamino)acridine (10) as the alternative reaction mechanism relevant under acidic conditions (Figure 2, Table 2). The attention was focused on the competition between 2-propanolate and hydroxyl anion, reactive species present in the reaction mixture. From the energy point of view it could be concluded that bulkiness of iso-propyl group has a significant influence. From kinetic as well thermodynamic point of view, it becomes evident that the reaction with 2-propanolate is disfavored, and should the bimolecular nucleophilic substitution take place, hydroxyl ion will be preferred as a nucleophile.

When sodium hydroxide was replaced by ammonium hydroxide, the product of the reaction was derivative $\mathbf{1 5}$ (Table 1) likely arising from intermolecular amine exchange via hemiaminal intermediate (Scheme 9). ${ }^{14,15}$

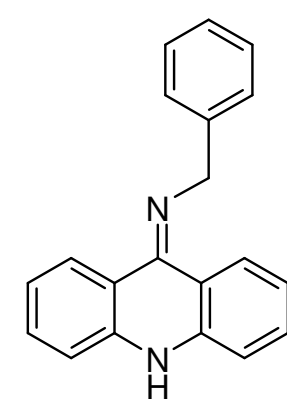

A

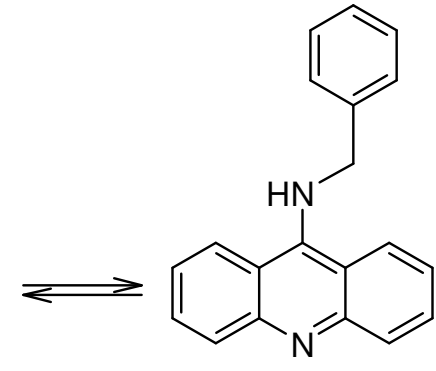

B

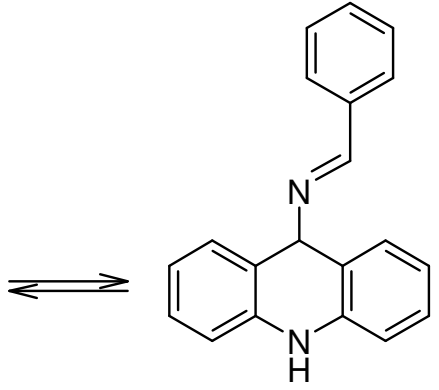

C

10

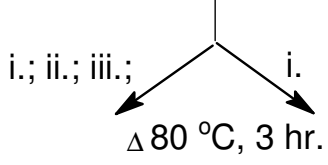<smiles>OCc1ccccc1</smiles>

12<smiles>Nc1c2ccccc2nc2ccccc12</smiles>

11<smiles>O=Cc1cccc(C(=O)c2cccc(C(=O)O)c2)c1</smiles>

13
14

i. $50 \% \mathrm{H}_{2} \mathrm{SO}_{4}$; ii. $50 \% \mathrm{H}_{3} \mathrm{PO}_{4}$; iii. $35 \% \mathrm{HCl}$

Scheme 6. Tautomeric forms of 9-(benzylamino)acridine (10) and their proposed reactions. 
<smiles></smiles>

i. the first step; ii. the second step;

Scheme 7. Proposed reaction mechanisms of the hydrolysis of derivative $\mathbf{1 0 .}$

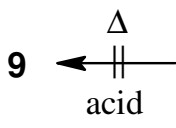

i: 2-propanol, $\mathrm{NaOH}, 80^{\circ} \mathrm{C}, 5 \mathrm{hr}$<smiles>Nc1ccc2c(NCc3ccccc3)c3ccc(N)cc3nc2c1</smiles>

$(n)=(2,3)$

Scheme 8. Synthesis of derivatives 8b-c.
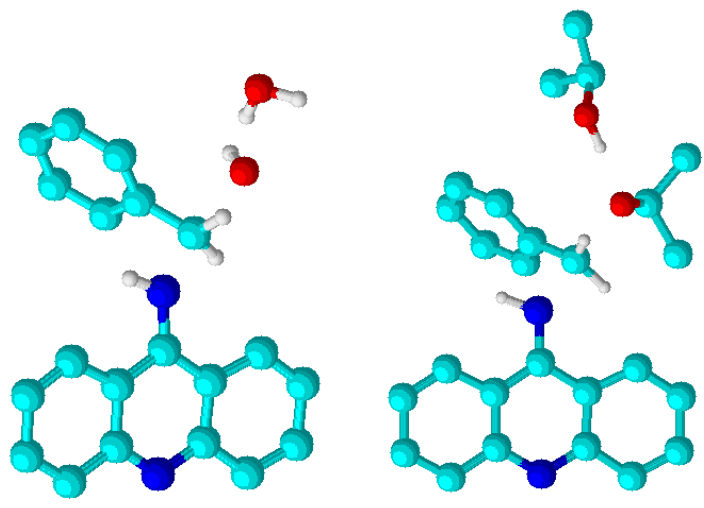

Figure 2. Plausible transition states (TS) for the theoretical reaction of derivative $\mathbf{1 0}$ with $O$ nucleophiles, hydroxyl (left) or 2-propanolate (right) ion. One molecule of the solvent was added to imitate the solvent effect. Hydrogens outside of the center of nucleophilic attack are omitted for clarity. Geometries of TS were obtained on semi-empirical level of theory using PM3 (MOPAC2012 ${ }^{16}$ ) (C - light blue, $\mathrm{N}$ - blue, $\mathrm{O}$ - red, $\mathrm{H}$ - white). 
<smiles>CCCC(=O)Nc1ccc2c(NCc3ccccc3)c3ccc(NC(=O)N(C)C)cc3nc2c1</smiles>

i. ex. $25 \% \underset{4}{\mathrm{NH}} \mathrm{OH}, \mathrm{MeOH}, 50^{\circ} \mathrm{C}, 5 \mathrm{hr}$., $83 \%$.

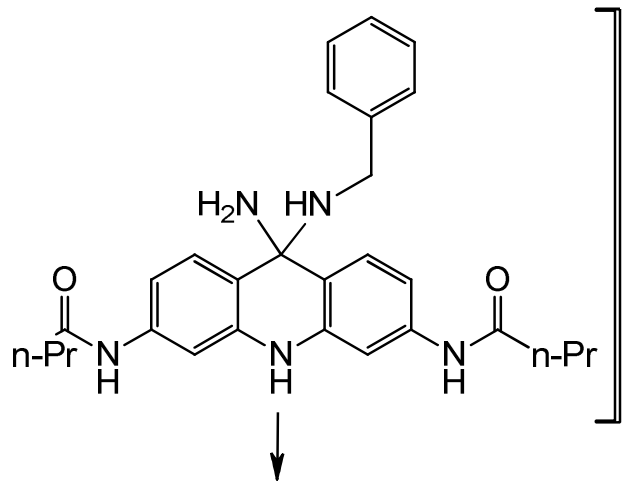<smiles>CCCC(=O)Nc1ccc2c(N)c3ccc(NC(=O)PC)cc3nc2c1</smiles>

15

Scheme 9. Intermolecular amine exchange of derivative 7a via hemiaminal intermediate.

Table 2. Energetic terms of the reaction of derivative $\mathbf{1 0}$ with $O$-nucleophiles

\begin{tabular}{|c|c|c|c|}
\hline \multirow[t]{2}{*}{ Reagents } & \multicolumn{3}{|c|}{ Energy $^{\mathrm{a}}$} \\
\hline & Reactants & $\begin{array}{c}\text { Transition } \\
\text { state }\end{array}$ & Products \\
\hline $\mathrm{b}$ & -24.18 & 7.47 & -52.89 \\
\hline \multirow[t]{3}{*}{$\mathrm{c}$} & -55.36 & -17.96 & -68.62 \\
\hline & \multicolumn{2}{|c|}{$\Delta \mathrm{E}^{\neq \mathrm{d}}$} & $\Delta \mathrm{E}^{\mathrm{e}}$ \\
\hline & TS-RCT & TS-PDT & PDT-RCT \\
\hline b & 31.65 & 60.36 & -28.71 \\
\hline $\mathrm{c}$ & 37.40 & 50.66 & -13.26 \\
\hline
\end{tabular}

${ }^{\mathrm{a}}$ Heat of formation (Kcal.mol ${ }^{-1}$ ) obtained by PM3 calculation.

${ }^{\mathrm{b}}$ Derivative $\mathbf{1 0}$ and hydroxyl ion.

${ }^{\mathrm{c}}$ Derivative 10 and 2-propanolate ion.

${ }^{\mathrm{d}}$ Energy gab between transition state (TS) and reactants (RCT) or transition state (TS) and products (PDT).

${ }^{\mathrm{e}}$ Energy gab between products (PDT) and reactants (RCT).

\section{Preparation of trisubstituted acridines}

Having resolved above mentioned problems, free amines 8a-c were utilized for the preparation of isothiocyanates 16a-c which might serve as key synthons in the synthesis of novel 3,6,9trisubstituted acridines. To overcome difficulties related to handling toxic and malodorous thiophosgene, there was decided to synthetize isothiocyanates $\mathbf{1 6 a - c}$ by the reaction of 
corresponding dithiocarbamates with thiophilic reagents, diacetoxyiodobenzene or cyanuric chloride. ${ }^{17-19}$ Unfortunatelly, the preparation of dithiocarbamates by the reaction of amines 8a-c with carbon disulfide was not successful, probably due to low nucleophilicity of amines 8a-c. Therefore the thiophosgene-mediated formation of isothiocyanates had to be used (Scheme 10).<smiles>CC(C)(C)Oc1ccc2c(NCc3ccccc3)c3ccc(N)cc3nc2c1</smiles><smiles>CN(C)CCNC(=O)Nc1ccc2c(NCc3ccccc3)c3ccc(NC(=O)NCCN(C)C)cc3nc2c1</smiles>

i. $\mathrm{CSCl}_{2}, \mathrm{NaHCO}_{3}, \mathrm{H}_{2} \mathrm{O}$, Acetone, $1 \mathrm{hr}$., $0^{\circ} \mathrm{C}$;

ii. $\left(\mathrm{CH}_{3}\right)_{2} \mathrm{~N}-\mathrm{CH}_{2} \mathrm{CH}_{2}-\mathrm{NH}_{2}$, Methanol, $1 \mathrm{hr}$., $0^{\circ} \mathrm{C}$; iii. MNO, Methanol, $1 \mathrm{hr}$., r.t. .<smiles></smiles>

ii.

$\nabla$<smiles>NCc1ccccc1</smiles><smiles>CNc1c2ccc(NC(=S)NCCN(C)C)cc2nc2cc(NC(=S)NCCN(C)C)ccc12</smiles>

$(n)=(1-3)$

Scheme 10. Preparation of trisubstituted acridines 17a-c and 18a-c.

An attempt to use two-phase system (dichloromethane/water), the standard routine in the synthesis of isothiocyanates, proved unsuccessful owing to low solubility of amines 8a-c. Luckily enough there was found, that amines 8a-c are soluble in mixtures of acetone or acetonitrile with water, while they are not in above mentioned individual solvents. This finding prompted us to find a suitable combination of reactants and solvents to obtain homogenous reaction mixture. The best choice turned out to be the combination of acetone/water in a ratio 7:1 as the solvent for amines 8a-c. The solution of amine was added dropwise to thiophosgenecontaining solution of sodium bicarbonate in acetone/water (1:3). Obtained isothiocyanates 16ac were not purified (expected low stability). Crude isothiocyanates 16a-c were reacted with $N, N$ dimethylethylenamine in methanol at $0{ }^{\circ} \mathrm{C}$. There should be pointed out that attempts to prepare 
thioureas 17a-c in commonly used organic solvents led to a mixture of unidentified products. As the reaction progressed, the resulting mixture became homogenous and the formation of corresponding thioureas 17a-c was observed by TLC analysis. Thioureas 17a-c were purified by gradient chromatography and then used in the reaction with mesityl nitriloxide providing ureas 18a-c.

\section{Conclusions}

We have developed a synthetic methodology for the preparation of 3,6- urea substituted 9[(phenylalkyl)amino]acridines as a prospective G-quadruplex stabilizing agents. We rationalized the unexpected results observed for some reactions described within the body of this paper and we proposed reasonable reaction mechanisms. We found reasonable reaction conditions for the transformation of poorly soluble amines 8a-c with low nucleophilicity into corresponding isothiocyanates 16a-c, key intermediates toward the desired products.

\section{Experimental Section}

General. All chemicals and reagents were reagent grade and were used without further purification. ${ }^{1} \mathrm{H}(400 \mathrm{MHz}, 600 \mathrm{MHz})$ and ${ }^{13} \mathrm{C}(100 \mathrm{MHz}, 150 \mathrm{MHz}) \mathrm{NMR}$ spectra were measured on a Varian Mercury Plus or a Varian VNMRS NMR spectrometers at room temperature in $\mathrm{CD}_{3} \mathrm{OD}$ or DMSO- $d_{6}$ using TMS as an internal standard ( 0 ppm for both nuclei). Melting points were determined with a Koffler hot-stage apparatus and are uncorrected. Elemental analyses were performed on a Perkin-Elmer analyzer CHN 2400. Reactions were monitored by thin-layer chromatography (TLC) using Silufol plates with detection at $254 \mathrm{~nm}$. Preparative column chromatography was conducted using Kiesegel Merck 60 column, type 9385 (grain size $250 \mathrm{~nm}$ ).

9-Benzylaminoacridine (10) and 9(10H)acridone (10a) were synthetized according to the published procedure, and all physical-chemical characteristic were in accordance to previously published results. ${ }^{20,21} 9$-Aminoacridine (11) was commercially available.

2,2',4,4'-Tetranitrodiphenylmethane (2). Performed according to the literature protocol. ${ }^{13}$ Cream solid, yield 84\%, mp 168-170 ${ }^{\circ} \mathrm{C} .{ }^{1} \mathrm{H}$ NMR (400 MHz, DMSO- $\left.d_{6}\right) \delta_{\mathrm{H}} 8.82$ (d, 2H, H-3,3', $J 2.4 \mathrm{~Hz}$ ), 8.49 (dd, 2H, H-5,5', J $8.6 \mathrm{~Hz}, J_{2} 2.4 \mathrm{~Hz}$ ), 7.60 (d, 2H, H-6,6', J 8.6 Hz), 4.79 (s, 2H, $\left.\mathrm{CH}_{2}\right) .{ }^{13} \mathrm{C}$ NMR $\left(100 \mathrm{MHz}, \mathrm{DMSO}-d_{6}\right) \delta_{\mathrm{C}} 149.02,147.05,140.13,134.10,128.23,120.82$, 35.47. Anal. Calcd for $\mathrm{C}_{13} \mathrm{H}_{8} \mathrm{~N}_{4} \mathrm{O}_{8}$ (348.22): C, 44.84; H, 2.32; N, 16.09\%. Found: C, 45.07; H, $2.25 ; \mathrm{N}, 16.29 \%$.

2,2',4,4' -Tetranitrodibenzophenone (3). $\mathrm{CrO}_{3}(17 \mathrm{~g}, 0.17 \mathrm{~mol}$ ) was slowly added (over $10 \mathrm{~min}$ period) to a refluxing solution of $2(10 \mathrm{~g}, 28.7 \mathrm{mmol})$ in $98 \%$ acetic acid $(50 \mathrm{~mL})$, and the mixture was refluxed for $3 \mathrm{hr}$. Once cooled to room temperature, the resultant mixture was 
poured into water $(200 \mathrm{~mL})$, the precipitated product was filtered off with suction, and was consecutively washed with water $(10 \mathrm{~mL})$, ethanol $(10 \mathrm{~mL})$ and diethyleter $(10 \mathrm{~mL})$ affording $9.8 \mathrm{~g}$ of product 3 as a pale yellow solid. Yield $94 \%$. mp $234-236{ }^{\circ} \mathrm{C} .{ }^{1} \mathrm{H} \mathrm{NMR}(400 \mathrm{MHz}$, DMSO- $\left.d_{6}\right) \delta_{\mathrm{H}} 8.95(2 \mathrm{H}, \mathrm{d}, \mathrm{H}-3,3, J 2.1 \mathrm{~Hz}), 8.64\left(2 \mathrm{H}, \mathrm{dd}, \mathrm{H}-5,5\right.$ ', $\left.J_{1} 8.5 \mathrm{~Hz}, J_{2} 2.1 \mathrm{~Hz}\right), 8.03$ $(2 \mathrm{H}, \mathrm{d}, \mathrm{H}-6,6$ ', $J 2.0 \mathrm{~Hz}) .{ }^{13} \mathrm{C}$ NMR (100 MHz, DMSO- $\left.d_{6}\right) \delta_{\mathrm{C}} 188.26,150.17,148.03,134.88$, 132.89, 128.73, 120.66. Anal. Calcd for $\mathrm{C}_{13} \mathrm{H}_{6} \mathrm{~N}_{4} \mathrm{O}_{9}$ (362.20): C, 43.11; H, 1.67; N, 15.47\%. Found: C, 43.32; H, 1.60; N, 15.32\%.

3,6-Diamino-9(10H)acridone (4). $\mathrm{SnCl}_{2} \cdot 2 \mathrm{H}_{2} \mathrm{O}(94 \mathrm{~g}, 0.417 \mathrm{~mol})$ was slowly added to a refluxing solution of $3(10 \mathrm{~g}, 27.6 \mathrm{mmol})$ in $35 \%$ hydrochloric acid $(150 \mathrm{~mL})$, followed by the addition of ethanol $(25 \mathrm{~mL})$. The mixture was heated for $18 \mathrm{hr}$ at $90{ }^{\circ} \mathrm{C}$. On cooling to room temperature, the precipitate was filtered off with suction, and was allowed to dry under air over night. Resulting solid was disolved in hot $0.1 \mathrm{M}$ hydrochloric acid (50 mL), and the solution was alkalized with $80 \% \mathrm{NaOH}$. On cooling, the precipitated product was filtered off with suction to give $5 \mathrm{~g}$ of the product 4 as a light red solid. Yield $80 \%, \mathrm{mp}>300{ }^{\circ} \mathrm{C}$. ${ }^{1} \mathrm{H} \mathrm{NMR}(400 \mathrm{MHz}$, DMSO- $\left.d_{6}\right): \delta_{\mathrm{H}} 10.61(1 \mathrm{H}, \mathrm{s}, \mathrm{NH}), 7.78\left(2 \mathrm{H}, \mathrm{d}, \mathrm{H}-1,8, J_{1} 8.4 \mathrm{~Hz}\right), 6.41\left(2 \mathrm{H}, \mathrm{dd}, \mathrm{H}-2,7, J_{1} 2.0 \mathrm{~Hz}\right.$, $\left.J_{2} 8.4 \mathrm{~Hz}\right), 6.34(2 \mathrm{H}, \mathrm{d}, \mathrm{H}-4,5, J 2.0 \mathrm{~Hz}), 5.86\left(4 \mathrm{H}, \mathrm{s}, 2 \times \mathrm{NH}_{2}\right) .{ }^{13} \mathrm{C}$ NMR $\left(100 \mathrm{MHz}, \mathrm{DMSO}-d_{6}\right)$ : $\delta_{\mathrm{C}} 174.68(\mathrm{CO}), 153.09$ (C3, C6), 143.60 (C4a, C10a), 127.72 (C1, C8), 112.26 (C8a, C9a), 110.49 (C2, C7), 96.33 (C4, C5). Anal. Calcd for $\mathrm{C}_{13} \mathrm{H}_{11} \mathrm{~N}_{3} \mathrm{O}$ (225.24): C, 69.32; H, 4.92; N, $18.66 \%$. Found: C, 69.55; H, 4.85; N, $18.93 \%$.

3,6-Di(butanoylamino)-9(10H)acridone (5). Diaminoacridone 4 (10 g, $44.4 \mathrm{mmol}$ ) was added to $70 \mathrm{~mL}$ of butyric anhydride $(0.428 \mathrm{~mol})$. The reaction mixture was heated at $100{ }^{\circ} \mathrm{C}$ while being stirred vigorously for $1.5 \mathrm{hr}$. Once the resulting mixture had cooled to room temperature, $30 \mathrm{~mL}$ of acetone was added. The mixture was poured into $200 \mathrm{~mL}$ of brine with an additive of $15 \mathrm{~mL}$ of saponate. A concentrated solution of sodium hydroxide was added to the mixture in 25 $\mathrm{mL}$ portions over $15 \mathrm{~min}$, until an alkaline $\mathrm{pH}$ had been achieved. The precipitated product was filtered off with suction. Then, product was crystallized from ethanol. Light brown solid, yield 49\%, $8 \mathrm{~g}, \mathrm{mp}>300{ }^{\circ} \mathrm{C} .{ }^{1} \mathrm{H}$ NMR (400 MHz, DMSO- $\left.d_{6}\right): \delta_{\mathrm{H}} 11.63(1 \mathrm{H}, \mathrm{s}, \mathrm{NH}), 10.25(2 \mathrm{H}, \mathrm{s}$, $2 \times \mathrm{NH}-\mathrm{CO}), 8.15(2 \mathrm{H}, \mathrm{s}, \mathrm{H}-4,5), 8.09(2 \mathrm{H}, \mathrm{d}, \mathrm{H}-1,8, J 8.4 \mathrm{~Hz}), 7.20(2 \mathrm{H}, \mathrm{d}, \mathrm{H}-2,7, J 8.4 \mathrm{~Hz})$, $2.37\left(4 \mathrm{H}, \mathrm{t}, 2 \times \mathrm{CH}_{2}-\mathrm{CO}, J 6.8 \mathrm{~Hz}\right), 1.68-1.63\left(4 \mathrm{H}, \mathrm{m}, 2 \times \mathrm{CH}_{2}\right), 0.95\left(6 \mathrm{H}, \mathrm{t}, 2 \times \mathrm{CH}_{3}, J 7.2 \mathrm{~Hz}\right) .{ }^{13} \mathrm{C}$ NMR (100 MHz, DMSO- $\left.d_{6}\right): \delta_{\mathrm{C}} 174.89$ (CO), 171.84 (2×NH-CO), 143.14 (C4a, C10a), 142.02 (C3, C6), 126.70 (C1, C8), 116.27 (C8a, C9a), 113.14 (C2, C7), 104.55 (C4, C5), $38.42\left(2 \times \mathrm{CH}_{2^{-}}\right.$ $\mathrm{CO}), 18.45\left(2 \times \mathrm{CH}_{2}\right), 13.55\left(2 \times \mathrm{CH}_{3}\right)$. Anal. Calcd for $\mathrm{C}_{21} \mathrm{H}_{23} \mathrm{~N}_{3} \mathrm{O}_{3}$ (365.43): C, 69.02; H, 6.34; N, $11.50 \%$. Found: C, 69.07; H, 6.23; N, 11.67\%.

3,6-Di(butanoylamino)-9-chloroacridine (6). Finely powdered acridone 5 (5 g, $13.7 \mathrm{mmol}$ ) was slowly added to $20 \mathrm{~mL}$ of $\mathrm{POCl}_{3}(0.214 \mathrm{~mol})$ over $15 \mathrm{~min}$. The mixture was heated at $70{ }^{\circ} \mathrm{C}$ while being stirred vigorously. After $90 \mathrm{~min}$, the resulting mixture was cooled to room temperature and slowly poured onto $100 \mathrm{~g}$ of ice in $100 \mathrm{~mL}$ of distilled water. A concentrated solution of sodium hydroxide was added to the resultant mixture in $25 \mathrm{~mL}$ portions in $15 \mathrm{~min}$ intervals until an alkaline $\mathrm{pH}$ had been achieved. The precipitated product was filtered off with suction, was washed with distilled water and was dried overnight. The resultant crude product 6 
was crystallized from ethyl acetate, this operation yielded $2.5 \mathrm{~g}$ as a light yellow solid. Yield 47\%, mp 153-155 ${ }^{\circ} \mathrm{C} .{ }^{1} \mathrm{H}$ NMR (400 MHz, DMSO- $\left.d_{6}\right): \delta_{\mathrm{H}} 10.45(2 \mathrm{H}, \mathrm{s}, 2 \times \mathrm{NH}-\mathrm{CO}), 8.58(2 \mathrm{H}, \mathrm{d}$, $\mathrm{H}-4,5, J 2.0 \mathrm{~Hz}), 8.27(2 \mathrm{H}, \mathrm{d}, \mathrm{H}-1,8, J 9.6 \mathrm{~Hz}), 7.76\left(2 \mathrm{H}, \mathrm{dd}, \mathrm{H}-2,7, J_{1} 2.0 \mathrm{~Hz}, J_{2} 9.6 \mathrm{~Hz}\right), 2.43$ $\left(4 \mathrm{H}, \mathrm{t}, 2 \times \mathrm{CH}_{2}-\mathrm{CO}, J 7.6 \mathrm{~Hz}\right), 1.72-1.68\left(4 \mathrm{H}, \mathrm{m}, 2 \times \mathrm{CH}_{2}\right), 0.98\left(6 \mathrm{H}, \mathrm{t}, 2 \times \mathrm{CH}_{3}, J 7.6 \mathrm{~Hz}\right) .{ }^{13} \mathrm{C}$ NMR (100 MHz, DMSO- $\left.d_{6}\right): \delta_{\mathrm{C}} 172.08$ (2×CO), 149.50 (C9), 141.24 (C4a, C10a), 139.13 (C3, C6), 124.81 (C1, C8), 121.69 (C2, C7), 119.34 (C8a, C9a), 113.58 (C4, C5), 38.46 (2×CH$\left.{ }_{2}-\mathrm{CO}\right)$, $18.34\left(2 \times \mathrm{CH}_{2}\right), 13.57\left(2 \times \mathrm{CH}_{3}\right)$. Anal. Calcd for $\mathrm{C}_{21} \mathrm{H}_{22} \mathrm{ClN}_{3} \mathrm{O}_{2}$ (383.87): C, 65.71; H, 5.78; N, $10.95 \%$. Found: C, 65.53; H, 5.87; N, 10.83\%.

General procedure for the preparation of 3,6-di(butanoylamino)-9-[(phenylalkyl)amino] acridine (7a-c). Chloroacridine $6(1.0 \mathrm{~g}, 2.6 \mathrm{mmol})$ was dissolved in hot acetonitrile $(60 \mathrm{~mL})$, followed by the addition of corresponding amine $(8.0 \mathrm{mmol})$. The mixture was refluxed for $1 \mathrm{hr}$. and the precipitated product was filtered off, was washed with acetonitrile. To remove the rest of the amine, the product was suspended in acetone, the mixture was vigorously stirred under reflux for $2 \mathrm{hr}$. On cooling, the product was filtered off, and was crystallized from ethanol to afford yellow crystals.

3,6-Di(butanoylamino)-9-(benzylamino)acridine hydrochloride (7a). Yellow crystalline solid, yield 77\%, $1 \mathrm{~g}, \mathrm{mp}>300{ }^{\circ} \mathrm{C} .{ }^{1} \mathrm{H}$ NMR $\left(600 \mathrm{MHz}, \mathrm{DMSO}-d_{6}\right)$ : $\delta_{\mathrm{H}} 13.31(1 \mathrm{H}, \mathrm{bs}, \mathrm{NH}-10), 10.84$ $(2 \mathrm{H}, \mathrm{s}, 2 \mathrm{xNHCO}), 10.12\left(1 \mathrm{H}, \mathrm{bs}, 9-\left(\mathrm{NHCH}_{2}\right)\right), 8.44-8.41(4 \mathrm{H}, \mathrm{m}, \mathrm{H}-1,8, \mathrm{H}-4,5), 7.46-7.43$ (4H, m, H-2'6', H-2,7), $7.40-7.37\left(2 \mathrm{H}, \mathrm{m}, \mathrm{H}-3^{\prime}, 5^{\prime}\right), 7.31-7.28(1 \mathrm{H}, \mathrm{m}, \mathrm{H}-4), 5.27(2 \mathrm{H}, \mathrm{s}$, $\left.\mathrm{NHCH}_{2} \mathrm{Ph}\right), 2.42\left(4 \mathrm{H}, \mathrm{t}, 2 \mathrm{xCOCH} \mathrm{CH}_{2}, \mathrm{~J} .2 \mathrm{~Hz}\right), 1.67-1.61\left(4 \mathrm{H}, \mathrm{m}, 2 \mathrm{x} \mathrm{CH}_{2} \mathrm{CH}_{3}\right), 0.92(6 \mathrm{H}, \mathrm{t}$, $\left.2 \mathrm{xCH}_{3}, J 7.2 \mathrm{~Hz}\right) .{ }^{13} \mathrm{C}$ NMR $\left(150 \mathrm{MHz}, \mathrm{DMSO}-d_{6}\right): \delta_{\mathrm{C}} 172.7(\mathrm{CO}), 155.4(\mathrm{C} 9), 144.4(\mathrm{C} 3, \mathrm{C} 6)$, 141.4 (C4a, C10a), 137.4 (C1'), 128.9 (C3', C5'), 127.6 (C4'), 126.8 (C1, C8), 126.7 (C2', C6'), 116.0 (C2, C7), 108.1 (C8a, C9a), 104.4 (C4, C5), $50.5\left(\mathrm{NH}_{\underline{C H}} \underline{2}_{2} \mathrm{Ph}\right), 38.5\left(\mathrm{COCH}_{2}\right), 18.4$ $\left(\mathrm{CH}_{2} \mathrm{CH}_{3}\right), 13.6\left(\mathrm{CH}_{3}\right)$. Anal. Calcd for $\mathrm{C}_{28} \mathrm{H}_{31} \mathrm{ClN}_{4} \mathrm{O}_{2}(491.02)$ : C, 68.49; H, 6.36; N, $11.41 \% \mathrm{~N}$. Found: C, 68.70; H, 6.45; N, 11.25\%.

3,6-Di(butanoylamino)-9-[(2-phenylethyl)amino]acridine hydrochloride (7b). Yellow crystalline solid, yield $76 \%, 1 \mathrm{~g}, \mathrm{mp}>300{ }^{\circ} \mathrm{C} .{ }^{1} \mathrm{H}$ NMR $\left(600 \mathrm{MHz}, \mathrm{DMSO}-d_{6}\right): \delta_{\mathrm{H}} 13.28(1 \mathrm{H}$, bs, NH-10), $10.85(2 \mathrm{H}, \mathrm{s}, 2 \mathrm{xNHCO}), 9.44\left(1 \mathrm{H}, \mathrm{bs}, 9-\left(\mathrm{NHCH}_{2}\right)\right), 8.50(2 \mathrm{H}, \mathrm{d}, \mathrm{H}-1,8, J \mathrm{~J} .0 \mathrm{~Hz})$, $8.42(2 \mathrm{H}, \mathrm{d}, \mathrm{H}-4,5, J 2.4 \mathrm{~Hz}), 7.52\left(2 \mathrm{H}, \mathrm{dd}, \mathrm{H}-2,7, J_{1} 9.0 \mathrm{~Hz}, J_{2} 2.4 \mathrm{~Hz}\right), 7.30-7.25$ (4H, m, H$\left.2^{\prime}, 6^{\prime}, \mathrm{H}-35^{\prime}\right), 7.20-7.17(1 \mathrm{H}, \mathrm{m}, \mathrm{H}-4), 4.27\left(2 \mathrm{H}, \mathrm{t}, \mathrm{NH} \underline{C H}_{2} \mathrm{CH}_{2} \mathrm{Ph}, J 7.2 \mathrm{~Hz}\right), 3.21(2 \mathrm{H}, \mathrm{t}$, $\left.\mathrm{NHCH}_{2} \underline{\mathrm{CH}}_{2} \mathrm{Ph}, J 7.2 \mathrm{~Hz}\right), 2.44\left(4 \mathrm{H}, \mathrm{t}, 2 \times \mathrm{COCH}_{2}, J 7.2 \mathrm{~Hz}\right), 1.70-1.63\left(4 \mathrm{H}, \mathrm{m}, 2 \mathrm{xCH}_{2} \mathrm{CH}_{3}\right)$, $0.95\left(6 \mathrm{H}, \mathrm{t}, 2 \mathrm{xCH}_{3}, J 7.2 \mathrm{~Hz}\right) .{ }^{13} \mathrm{C} \mathrm{NMR}\left(150 \mathrm{MHz}, \mathrm{DMSO}-d_{6}\right): \delta_{\mathrm{C}} 172.6$ (CO), $155.4(\mathrm{C} 9)$, 144.4 (C3, C6), 141.3 (C4a, C10a), 138.2 (C1'), 128.8 (C2', C6'), 128.4 (C3', C5'), 126.8 (C1, C8), 126.5 (C4), 115.9 (C2, C7), 108.1 (C8a, C9a), 104.3 (C4, C5), $50.0\left(\mathrm{NHCH}_{2} \mathrm{CH}_{2} \mathrm{Ph}\right), 38.5$ $\left(\mathrm{COCH}_{2}\right), 35.0\left(\mathrm{NHCH}_{2} \underline{\mathrm{CH}}_{2} \mathrm{Ph}\right), 18.4\left(\mathrm{CH}_{2} \mathrm{CH}_{3}\right), 13.6\left(\mathrm{CH}_{3}\right)$. Anal. Calcd for $\mathrm{C}_{29} \mathrm{H}_{33} \mathrm{ClN}_{4} \mathrm{O}_{2}$ (505.05): C, 68.97; H, 6.59; N, 11.09\%. Found: C, 68.78; H, 6.70; N, 11.26\%.

3,6-Di(butanoylamino)-9-[(3-phenylpropyl)amino]acridine hydrochloride (7c). Yellow crystalline solid, yield 74\%, $1 \mathrm{~g}, \mathrm{mp} 293-295{ }^{\circ} \mathrm{C} .{ }^{1} \mathrm{H}$ NMR $\left(600 \mathrm{MHz}, \mathrm{DMSO}-d_{6}\right): \delta_{\mathrm{H}} 13.22(1 \mathrm{H}$, bs, $N H-10), 10.88(2 \mathrm{H}, \mathrm{s}, 2 \mathrm{x} N \mathrm{CO}), 9.48\left(1 \mathrm{H}, \mathrm{bs}, 9-\left(\mathrm{NHCH}_{2}\right)\right), 8.39-8.39(4 \mathrm{H}, \mathrm{m}, \mathrm{H}-1,8, \mathrm{H}-$ 
4,5), 7.46 (2H, d, H-2,7, J 9.0 Hz), 7.25 - 7.22 (2H, m, H-3, 5'), 7.19 - 7.18 (2H, m, H-2',6), 7.15 - $7.13(1 \mathrm{H}, \mathrm{m}, \mathrm{H}-4), 4.00-3.97\left(2 \mathrm{H}, \mathrm{m}, \mathrm{NH} \underline{\mathrm{CH}_{2}} \mathrm{CH}_{2} \mathrm{CH}_{2} \mathrm{Ph}\right), 2.69$ (2H, t, $\left.\mathrm{NHCH}_{2} \mathrm{CH}_{2} \mathrm{CH}_{2} \mathrm{Ph}, J 7.2 \mathrm{~Hz}\right), 2.43\left(4 \mathrm{H}, \mathrm{t}, 2 \mathrm{CCOCH}_{2}, J 7.2 \mathrm{~Hz}\right), 2.20-2.15(2 \mathrm{H}, \mathrm{m}$, $\left.\mathrm{NHCH}_{2} \underline{\mathrm{CH}}_{2} \mathrm{CH}_{2} \mathrm{Ph}\right), 1.68-1.61\left(4 \mathrm{H}, \mathrm{m}, 2 \mathrm{XCH}_{2} \mathrm{CH}_{3}\right), 0.93\left(6 \mathrm{H}, \mathrm{t}, 2 \mathrm{xCH}_{3}, J 7.2 \mathrm{~Hz}\right) .{ }^{13} \mathrm{C} \mathrm{NMR}$ (150 MHz, DMSO-d $d_{6}$ : $\delta_{\mathrm{C}} 172.6$ (CO), 155.1 (C9), 144.3 (C3, C6), 141.2 (C4a, C10a), 140.9 (C1'), 128.3 (C2', C6', C3', C5'), 126.7 (C1, C8), 125.9 (C4'), 115.8 (C2, C7), 107.9 (C8a, C9a), 104.3 (C4, C5), $47.7\left(\mathrm{NH}_{\mathrm{CH}_{2}} \mathrm{CH}_{2} \mathrm{CH}_{2} \mathrm{Ph}\right), 38.5\left(\mathrm{COCH}_{2}\right), 32.23\left(\mathrm{NHCH}_{2} \mathrm{CH}_{2} \mathrm{CH}_{2} \mathrm{Ph}\right), 30.49$ $\left(\mathrm{NHCH}_{2} \underline{\mathrm{CH}}_{2} \mathrm{CH}_{2} \mathrm{Ph}\right), 18.4\left(\mathrm{CH}_{2} \mathrm{CH}_{3}\right), 13.6\left(\mathrm{CH}_{3}\right)$. Anal. Calcd for $\mathrm{C}_{30} \mathrm{H}_{35} \mathrm{ClN}_{4} \mathrm{O}_{2}$ (519.07): C, $69.42 ; \mathrm{H}, 6.80 \mathrm{~N}, 10.79 \%$. Found: C, 69.23; H, 6.58; N, 10.50\%.

General procedure for the preparation of 3,6-diamino-9-[(phenylalkyl)amino])acridine (8ac)

Derivatives 7a-c $(1.0 \mathrm{mmol})$ were dissolved in 2-propanol $(25 \mathrm{~mL})$, followed by the addition of $0.05 \mathrm{~mL}$ of concentrated sodium hydroxide solution. The reaction mixture was heated at $80{ }^{\circ} \mathrm{C}$ for $5 \mathrm{hr}$. The resulting mixture was then diluted with $20 \mathrm{~mL}$ of water, and was poured into 300 $\mathrm{mL}$ of water. $\mathrm{NaCl}$ was slowly added into the aqueous solution to precipitate the product. Products $8 \mathbf{a}, \mathbf{8 b}$ were filtered off as a solid. The product $8 \mathbf{c}$ deposited as an oil on the flask walls. Products 8a-c were crystallized from a mixture MeOH/THF to afford yellow crystals.

3,6-Diamino-9-(benzylamino)acridine (8a). Yellow crystalline solid, yield 80\%, $0.25 \mathrm{~g}, \mathrm{mp}$ 170-172 ${ }^{\circ} \mathrm{C} .{ }^{1} \mathrm{H}$ NMR $\left(600 \mathrm{MHz}, \mathrm{DMSO}-d_{6}\right): \delta_{\mathrm{H}} 8.04(2 \mathrm{H}, \mathrm{d}, \mathrm{H}-1,8, J 9.0 \mathrm{~Hz}), 7.40-7.39(2 \mathrm{H}$, m, H-2',6'), 7.37 - 7.34 (2H, m, H-3',5), $7.27-7.25$ (1H, m, H-4), $6.61-6.58$ (4H, m, H-2,7, $\mathrm{H}-4,5), 6.39\left(4 \mathrm{H}, \mathrm{bs}, 2 \mathrm{xNH}_{2}\right) 5.02\left(2 \mathrm{H}, \mathrm{s}, \mathrm{NH} \underline{C H}_{2} \mathrm{Ph}\right) .{ }^{13} \mathrm{C} \mathrm{NMR}\left(150 \mathrm{MHz}, \mathrm{DMSO}-d_{6}\right): \delta_{\mathrm{C}}$ 153.5 (C9), 152.9 (C3, C6), 144.1 (C4a, C10a), 139.1 (C1'), 128.6 (C3', C5'), 127.1 (C4'), 126.7 (C1, C8, C2', C6'), 113.4 (C2, C7), 104.9 (C8a, C9a), 96.7 (C4, C5), 51.1 ( $\left.\mathrm{NH}_{\underline{C H}}{ }_{2} \mathrm{Ph}\right)$. Anal. Calcd for $\mathrm{C}_{20} \mathrm{H}_{18} \mathrm{~N}_{4}$ (314.39): C, 76.41; H, 5.77; N, 17.82\%. Found: C, 76.61; H, 5.84; N, $17.96 \%$.

3,6-Diamino-9-[(2-phenylethyl)amino]acridine (8b). Yellow crystalline solid, yield 73\%, 0.24 g, mp 165-167 ${ }^{\circ} \mathrm{C} .{ }^{1} \mathrm{H}$ NMR $\left(600 \mathrm{MHz}, \mathrm{DMSO}-d_{6}\right): \delta_{\mathrm{H}} 8.08(2 \mathrm{H}, \mathrm{d}, \mathrm{H}-1,8, J 9.0 \mathrm{~Hz}), 7.28-$ $7.24\left(4 \mathrm{H}, \mathrm{m}, \mathrm{H}-2{ }^{\prime}, 6^{\prime}, \mathrm{H}-3^{\prime}, 5^{\prime}\right), 7.19-7.16(1 \mathrm{H}, \mathrm{m}, \mathrm{H}-4), 6.69\left(2 \mathrm{H}, \mathrm{dd}, \mathrm{H}-2,7, J_{1} 9.0 \mathrm{~Hz}, J_{2} 2.4\right.$ $\mathrm{Hz}), 6.62\left(4 \mathrm{H}, \mathrm{bs}, 2 \mathrm{xNH}_{2}\right), 6.59(2 \mathrm{H}, \mathrm{d}, \mathrm{H}-4,5, J 2.4 \mathrm{~Hz}), 4.08\left(2 \mathrm{H}, \mathrm{t}, \mathrm{NHC} \underline{C H}_{2} \mathrm{CH}_{2} \mathrm{Ph}, J 7.2 \mathrm{~Hz}\right)$, $3.10\left(2 \mathrm{H}, \mathrm{t}, \mathrm{NHCH}_{2} \underline{\mathrm{CH}}_{2} \mathrm{Ph}, J 7.2 \mathrm{~Hz}\right) .{ }^{13} \mathrm{C} \mathrm{NMR}\left(150 \mathrm{MHz}, \mathrm{DMSO}-d_{6}\right): \delta_{\mathrm{C}} 154.1(\mathrm{C} 9), 153.8$ (C3, C6), 142.6 (C4a, C10a), 138.5 (C1'), 128.8 (C2', C6'), 128.4 (C3', C5'), 127.0 (C1, C8) 126.4 (C4), 113.4 (C2, C7), 103.7 (C8a, C9a), 95.1 (C4, C5), $50.0 \quad\left(\mathrm{NH}_{2} \underline{H}_{2} \mathrm{CH}_{2} \mathrm{Ph}\right), 35.4$ $\left(\mathrm{NHCH}_{2} \underline{\mathrm{CH}}_{2} \mathrm{Ph}\right)$. Anal. Calcd for $\mathrm{C}_{21} \mathrm{H}_{20} \mathrm{~N}_{4}$ (328.41): C, 76.80; H, 6.14; N, 17.06\%. Found: C, $76.06 ; \mathrm{H}, 6.20 ; \mathrm{N}, 17.19 \%$.

3,6-Diamino-9-[(3-phenylpropyl)amino]acridine (8c). Yellow crystalline solid, yield 53\%, $0.18 \mathrm{~g}, \mathrm{mp} 155-157{ }^{\circ} \mathrm{C} .{ }^{1} \mathrm{H}$ NMR $\left(600 \mathrm{MHz}, \mathrm{DMSO}-d_{6}\right): \delta_{\mathrm{H}} 8.04(2 \mathrm{H}, \mathrm{d}, \mathrm{H}-1,8, J 9.0 \mathrm{~Hz}), 7.25$ - $7.23\left(2 \mathrm{H}, \mathrm{m}, \mathrm{H}-3^{\prime}, 5^{\prime}\right), 7.17$ - 7.14 (3H, m, H-2',6', H-4'), 6.67 - 6.65 (4H, m, H-2,7, H-4,5), $6.58\left(4 \mathrm{H}, \mathrm{bs}, 2 \mathrm{xNH}_{2}\right), 3.83-3.80\left(2 \mathrm{H}, \mathrm{m}, \mathrm{NH} \underline{\mathrm{CH}_{2}} \mathrm{CH}_{2} \mathrm{CH}_{2} \mathrm{Ph}\right), 2.65\left(2 \mathrm{H}, \mathrm{t}, \mathrm{NHCH}_{2} \mathrm{CH}_{2} \underline{C H}_{2} \mathrm{Ph}\right.$, $J 7.2 \mathrm{~Hz}), 2.10-2.05\left(2 \mathrm{H}, \mathrm{m}, \mathrm{NHCH}_{2} \underline{\mathrm{CH}}_{2} \mathrm{CH}_{2} \mathrm{Ph}\right) .{ }^{13} \mathrm{C} \mathrm{NMR}\left(150 \mathrm{MHz}, \mathrm{DMSO}-d_{6}\right): \delta_{\mathrm{C}} 153.96$ 
(C9), 153.55 (C3, C6), 142.99 (C4a, C10a), 141.12 (C1'), 128.31 (C3', C5'), 128.29 (C2', C6'), 126.91 (C1, C8), 125.86 (C4), 113.38 (C2, C7), 103.84 (C8a, C9a), 95.52 (C4, C5), 47.83 $\left(\mathrm{NH}_{\underline{C H}} \mathrm{CH}_{2} \mathrm{CH}_{2} \mathrm{Ph}\right), 32.28\left(\mathrm{NHCH}_{2} \mathrm{CH}_{2} \underline{\mathrm{CH}}_{2} \mathrm{Ph}\right), 31.22\left(\mathrm{NHCH}_{2} \underline{\mathrm{CH}}_{2} \mathrm{CH}_{2} \mathrm{Ph}\right)$. Anal. Calcd for $\mathrm{C}_{22} \mathrm{H}_{22} \mathrm{~N}_{4}$ (342.47): C, 77.16; H, 6.48; N, 16.36\%. Found: C, 76.97; H, 6.59; N, 16.27\%.

3,6,9-Triaminoacridine (9). Derivative 7a $(0.10 \mathrm{~g}, 0.2 \mathrm{mmol})$ was dissolved in $3 \mathrm{~mL}$ of $70 \%$ sulfuric acid, the mixture was vigorously stirred at $80{ }^{\circ} \mathrm{C}$ for $3 \mathrm{hr}$. The solution was then poured into ice water, was alkalized with sodium hydroxide, and the precipitate was filtered off. The product was dried in vacuo over $\mathrm{P}_{2} \mathrm{O}_{5}$ affording yellow solid. Yellow solid, yield 80\%, $0.036 \mathrm{~g}$, $\mathrm{mp}>300{ }^{\circ} \mathrm{C} .{ }^{1} \mathrm{H}$ NMR $\left(600 \mathrm{MHz}, \mathrm{DMSO}-d_{6}\right): \delta_{\mathrm{H}} 11.90\left(2 \mathrm{H}, \mathrm{s}, \mathrm{NH}_{2}-9\right), 8.49\left(4 \mathrm{H}, \mathrm{s}, \mathrm{NH}_{2}-3,6\right)$, $8.05(2 \mathrm{H}, \mathrm{d}, \mathrm{H}-1,8, J 9.0 \mathrm{~Hz}), 6.69(2 \mathrm{H}, \mathrm{d}, \mathrm{H}-2,7, J 9.0 \mathrm{~Hz}), 6.50(2 \mathrm{H}, \mathrm{s}, \mathrm{H}-4,5) .{ }^{13} \mathrm{C}$ NMR $(150$ MHz, DMSO- $d_{6}$ ): $\delta_{\mathrm{C}} 154.5$ (C9), 154.2 (C4a, C10a), 142.5 (C3, C6), 126.4 (C1, C8), 114.2 (C2, C7), 102.8 (C8a, C9a), 95.7 (C4, C5). Anal. Calcd for $\mathrm{C}_{13} \mathrm{H}_{12} \mathrm{~N}_{4}$ (224.26): C, 69.62; H, 5.39; N, 24.98\%. Found: C, 69.90; H, 5.49; N, 25.27\%.

3,6-Di(butanoylamino)-9-aminoacridine (15). Derivative 7a $(0.20 \mathrm{~g}, 0.4 \mathrm{mmol})$ was dissolved in methanol $(3 \mathrm{~mL})$, followed by the addition of $1 \mathrm{~mL}$ of concentrated amonnium hydroxide solution. The reaction mixture was heated at $50{ }^{\circ} \mathrm{C}$ for $5 \mathrm{hr}$. The resulting mixture was then poured into $30 \mathrm{~mL}$ of brine, and precipitated product was filtered off. Product $\mathbf{1 0}$ was crystallized from $\mathrm{MeOH}$ and dried in vacuo over $\mathrm{P}_{2} \mathrm{O}_{5}$ affording yellow crystals. Yellow crystalline solid, yield 83\%, $0.12 \mathrm{~g}, \mathrm{mp}>300{ }^{\circ} \mathrm{C} .{ }^{1} \mathrm{H}$ NMR (600 MHz, DMSO- $\left.d_{6}\right): \delta_{\mathrm{H}} 10.13$ (2H, s, 2xNHCO), $8.26(2 \mathrm{H}, \mathrm{d}, \mathrm{H}-1,8, J 9.0 \mathrm{~Hz}), 8.14(2 \mathrm{H}, \mathrm{s}, \mathrm{H}-4,5), 7.40\left(2 \mathrm{H}, \mathrm{dd}, \mathrm{H}-4,5, J_{1} 9.0 \mathrm{~Hz}, J_{2} 1.8 \mathrm{~Hz}\right)$, $2.37\left(4 \mathrm{H}, \mathrm{t}, 2 \mathrm{xCO} \underline{C H}_{2}, J 7.2 \mathrm{~Hz}\right), 1.68-1.62\left(4 \mathrm{H}, \mathrm{m}, 2 \underline{\mathrm{CH}}_{2} \mathrm{CH}_{3}\right), 0.95\left(6 \mathrm{H}, \mathrm{t}, 2 \mathrm{xCH}_{3}, J 7.2 \mathrm{~Hz}\right)$. ${ }^{13} \mathrm{C}$ NMR (150 MHz, DMSO- $\left.d_{6}\right): \delta_{\mathrm{C}} 171.7$ (CO), 149.6 (C9, C4a, C10a), 140.7 (C3, C6), 123.9 (C1, C8), 115.5 (C2, C7), 113.7 (C8a, C9a), 109.1 (C4, C5), $38.5\left(\mathrm{COCH}_{2}\right), 18.5\left(\underline{\mathrm{CH}}_{2} \mathrm{CH}_{3}\right)$, $13.6\left(\mathrm{CH}_{3}\right)$. Anal. Calcd for $\mathrm{C}_{21} \mathrm{H}_{24} \mathrm{~N}_{4} \mathrm{O}_{2}$ (364.44): C, 69.21; H, 6.64; N, 15.37\%. Found: C, $69.01 ; \mathrm{H}, 6.68 ; \mathrm{N}, 15.53 \%$.

General procedure for the preparation of 3,6-bis(isothiocyanato)-9-[(phenylalkyl) amino]acridine (16a-c). Diaminoacridines 8a-c $(1.6 \mathrm{mmol})$ were dissolved in a mixture acetone/water (10 $\mathrm{mL} ; 7: 1, \mathrm{v}: \mathrm{v})$. Resulting solution of amines were added dropwise to the vigorously stirred suspension of thiophosgene $(0.368 \mathrm{~mL}, 4.8 \mathrm{mmol})$ in a mixture of water/acetone $(10 \mathrm{~mL} ; 3: 1 ; \mathrm{v}: \mathrm{v})$ containing sodium bicarbonate $(1.34 \mathrm{~g}, 16 \mathrm{mmol}$, $)$ at $0{ }^{\circ} \mathrm{C}$, leading to the formation of isothiocyanates 16a-c as a yellow paste. After the addition was completed, the mixture was stirred for additional $30 \mathrm{~min}$. The crude product was filtered off with suction, was washed with acetone, and was dried in vacuo over $\mathrm{P}_{2} \mathrm{O}_{5}$ for $1 \mathrm{hr}$. Due to low stability, isothiocyanates 16a-c were used immediately for the preparation of thioureas $\mathbf{1 7 a - c .}$

General procedure for the preparation of 3,6-bis\{3-[2-(dimethylamino)ethyl]thioureido\}-9[(phenylalkyl)amino]acridine (17a-c). Previously prepared isothiocyanates 16a-c were suspended in methanol, followed by the addition of dimethylethylenediamine $(0.435 \mathrm{~mL}, 4$ mmol). The reaction mixtures were stirred at $0{ }^{\circ} \mathrm{C}$ for $1 \mathrm{hr}$, clear solutions were obtained at the 
end of the reaction. Subsequently, the solvent was evaporated under reduce pressure providing light brown oily residue. The crude product was purified on a silica gel column by gradient elution using a mixture acetone and $12 \%$ aqueous solution of diethyl amine (from 15:1 to 5:1, $\mathrm{v} / \mathrm{v})$. Evaporation of the solvent under reduced pressure provided products 17a-c as a dark yellow semi-solid residues.

3,6-Bis\{3-[2-(dimethylamino)ethyl]thioureido\}-9-(benzylamino)acridine $\quad$ (17a). Yield (referred to 8a) 48\%, 0.44 g. ${ }^{1} \mathrm{H}$ NMR (400 MHz, $\left.\mathrm{CD}_{3} \mathrm{OD}\right): \delta_{\mathrm{H}} 7.87(2 \mathrm{H}, \mathrm{s}, \mathrm{H}-4,5), 7.74(2 \mathrm{H}, \mathrm{d}$, $\mathrm{H}-1,8, J 9.2 \mathrm{~Hz}), 7.31$ - 7.27 (4H, m, H-2',6', H-3',5), $7.24-7.19$ (3H, m, H-2,7, H-4), 4.90

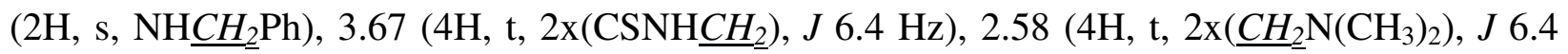
$\mathrm{Hz}), 2.27\left(12 \mathrm{H}, \mathrm{s}, 4 \mathrm{xCH} \mathrm{CH}_{3}\right) .{ }^{13} \mathrm{C} \mathrm{NMR}\left(100 \mathrm{MHz}, \mathrm{CD}_{3} \mathrm{OD}\right): \delta_{\mathrm{C}} 181.7$ (CS), 155.5 (C9), 145.9 (C3, C6), 143.6 (C4a, C10a), 138.9 (C1'), 130.1 (C3', C5'), 128.8 (C4'), 127.9 (C2', C6'), 126.1 (C1,

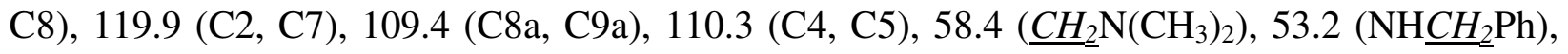
$45.6\left(\mathrm{CH}_{3}\right), 42.6\left(\mathrm{CSNH} \underline{C H}_{2}\right)$. Anal. Calcd for $\mathrm{C}_{30} \mathrm{H}_{38} \mathrm{~N}_{8} \mathrm{~S}_{2}(574.80)$ : C, 62.69; H, 6.66; N, $19.49 \%$ N. Found: C, 62.47; H, 6.58 N, 19.70\%.

3,6-Bis\{3-[2-(dimethylamino)ethyl]thioureido\}-9-[(2-phenylethyl)amino]acridine (17b). Yield (referred to 8b) 43\%, 0.40 g. ${ }^{1} \mathrm{H}$ NMR (400 MHz, $\left.\mathrm{CD}_{3} \mathrm{OD}\right): \delta_{\mathrm{H}} 7.96(2 \mathrm{H}, \mathrm{d}, \mathrm{H}-1,8, J 9.2$ $\mathrm{Hz}), 7.87$ (2H, s, H-4,5), 7.23 - 7.13 (7H, m, H-2,7, H-2'6, H-3',5', H-4), 3.98 (2H, t, $\left.\mathrm{NH} \underline{C H}_{2} \mathrm{CH}_{2} \mathrm{Ph}, J 7.2 \mathrm{~Hz}\right), 3.74(4 \mathrm{H}, \mathrm{t}, 2 \mathrm{x}(\mathrm{CSNH} \underline{\mathrm{CH}} \underline{2}), J 6.4 \mathrm{~Hz}), 3.01\left(2 \mathrm{H}, \mathrm{t}, \mathrm{NHCH}_{2} \underline{\mathrm{CH}}_{2} \mathrm{Ph}, J\right.$ $7.2 \mathrm{~Hz}), 2.61\left(4 \mathrm{H}, \mathrm{t}, 2 \mathrm{x}\left(\underline{\mathrm{CH}}_{2} \mathrm{~N}\left(\mathrm{CH}_{3}\right)_{2}\right), J 6.4 \mathrm{~Hz}\right), 2.31\left(12 \mathrm{H}, \mathrm{s}, 4 \mathrm{xCH}_{3}\right) .{ }^{13} \mathrm{C} \mathrm{NMR}(100 \mathrm{MHz}$, $\left.\mathrm{CD}_{3} \mathrm{OD}\right): \delta_{\mathrm{C}} 182.2(\mathrm{CS}), 153.4(\mathrm{C} 9), 149.9$ (C3, C6), 143.1 (C4a, C10a), 139.8 (C1'), 129.9 (C2', C6'), 129.6 (C3', C5'), 127.6 (C4), 125.7 (C1, C8), 120.3 (C2, C7), 116.9 (C4, C5), 113.7 (C8a, C9a), $58.7\left(\underline{\mathrm{CH}}_{2} \mathrm{~N}\left(\mathrm{CH}_{3}\right)_{2}\right), \quad 52.1 \quad\left(\mathrm{NH}_{\mathrm{CH}_{2}} \mathrm{CH}_{2} \mathrm{Ph}\right), 45.6 \quad\left(\mathrm{CH}_{3}\right), 43.2 \quad\left(\mathrm{CSNH} \underline{C H}_{2}\right), 37.9$ $\left(\mathrm{NHCH}_{2} \underline{\mathrm{CH}}_{2} \mathrm{Ph}\right)$. Anal. Calcd for $\mathrm{C}_{31} \mathrm{H}_{40} \mathrm{~N}_{8} \mathrm{~S}_{2}$ (588.83): C, 63.23; H, 6.85; N, 19.03\%. Found: C, $63.05 ; \mathrm{H}, 6.76 ; \mathrm{N}, 18.78 \%$.

3,6-Bis\{3-[2-(dimethylamino)ethyl]thioureido\}-9-[(3-phenylpropyl)amino]acridine (17c). Yield (referred to 8c) 38\%, 0.38 g. ${ }^{1} \mathrm{H}$ NMR (400 MHz, $\left.\mathrm{CD}_{3} \mathrm{OD}\right): \delta_{\mathrm{H}} 7.97(2 \mathrm{H}, \mathrm{d}, \mathrm{H}-1,8, J 9.2$ Hz), 7.87 (2H, s, H-4,5), 7.22 - 7.18 (2H, m, H-3, 5'), 7.14 - 7.08 (5H, m, H-2,7, H-2',6' H-4'), $3.76-3.72\left(2 \mathrm{H}, \mathrm{m}, \mathrm{NH} \underline{\mathrm{CH}}{ }_{2} \mathrm{CH}_{2} \mathrm{CH}_{2} \mathrm{Ph}\right), 3.34-3.30\left(4 \mathrm{H}, \mathrm{t}, 2 \mathrm{x}\left(\mathrm{CSNH} \underline{C H}_{2}\right), J 6.4 \mathrm{~Hz}\right), 2.68-$ $2.60\left(6 \mathrm{H}, \mathrm{m}, \mathrm{NHCH}_{2} \mathrm{CH}_{2} \underline{\mathrm{CH}}_{2} \mathrm{Ph}, 2 \mathrm{x}\left(\underline{\mathrm{CH}}_{2} \mathrm{~N}\left(\mathrm{CH}_{3}\right)_{2}\right)\right), 2.31(12 \mathrm{H}, \mathrm{s}, 4 \mathrm{xCH}), 2.09-2.02(2 \mathrm{H}, \mathrm{m}$, $\mathrm{NHCH}_{2} \underline{\mathrm{CH}}_{2} \mathrm{CH}_{2} \mathrm{Ph}$ ). ${ }^{13} \mathrm{C} \mathrm{NMR}\left(100 \mathrm{MHz}, \mathrm{CD}_{3} \mathrm{OD}\right): \delta_{\mathrm{C}} 182.2$ (CS), 153.5 (C9), 150.1 (C3, C6), 143.1 (C4a, C10a), 142.5 (C1'), 129.5 (C3', C5', C2', C6'), 127.1 (C4), 125.7 (C1, C8), 120.1 (C2,

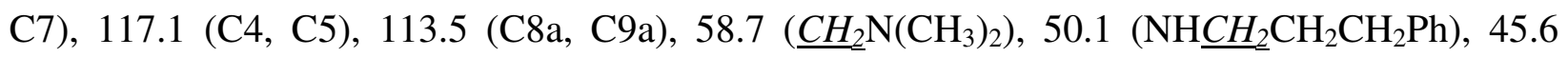
$\left(\mathrm{CH}_{3}\right), 43.2\left(\mathrm{CSNH}_{\underline{C H}}\right), 34.0\left(\mathrm{NHCH}_{2} \mathrm{CH}_{2} \underline{\mathrm{CH}}_{2} \mathrm{Ph}\right), 33.5\left(\mathrm{NHCH}_{2} \underline{\mathrm{CH}}_{2} \mathrm{CH}_{2} \mathrm{Ph}\right)$. Anal. Calcd for $\mathrm{C}_{32} \mathrm{H}_{42} \mathrm{~N}_{8} \mathrm{~S}_{2}$ (602.85): C, 63.75; H, 7.02 N, 18.59\%. Found: C, 63.90; H, 7.13; N, 18.77\%.

General procedure for the preparation of 3,6-bis $\{3-[2-($ dimethylamino)ethyl]ureido\}-9[(phenylalkyl)amino]acridine (18a-c). Previously prepared thioureas 17a-c $(0.9 \mathrm{mmol})$ were disolved in methanol $(20 \mathrm{~mL})$, followed by the addition of mesityl nitriloxide $(0.4 \mathrm{~g}, 2.5 \mathrm{mmol})$. The reaction mixtures were stirred for $1 \mathrm{hr}$ at room temperature. Subsequently, the solvent was evaporated under reduce pressure providing light brown oily residue. The crude product was 
purified on a silica gel column by gradient elution using a mixture acetone and $12 \%$ aqueous solution of diethyl amine (from 15:1 to 5:1, v/v). Evaporation of the solvent under reduced pressure provided product $\mathbf{1 8 a - c}$ as a yellow semi-solid residues.

3,6-Bis\{3-[2-(dimethylamino)ethyl]ureido\}-9-(benzylamino)acridine (18a). Yield 49\%, 0.24 g. ${ }^{1} \mathrm{H}$ NMR (600 MHz, CD $\left.{ }_{3} \mathrm{OD}\right): \delta_{\mathrm{H}} 7.98(2 \mathrm{H}, \mathrm{d}, \mathrm{H}-1,8, J 9.6 \mathrm{~Hz}), 7.81(2 \mathrm{H}, \mathrm{s}, \mathrm{H}-4,5), 7.39-$ $7.38\left(4 \mathrm{H}, \mathrm{m}, \mathrm{H}-2{ }^{\prime}, 6^{\prime}, \mathrm{H}-3^{\prime}, 5^{\prime}\right), 7.31-7.28(1 \mathrm{H}, \mathrm{m}, \mathrm{H}-4), 7.10\left(2 \mathrm{H}, \mathrm{dd}, \mathrm{H}-2,7, J_{1} 9.6 \mathrm{~Hz}, J_{2} 1.8\right.$ $\mathrm{Hz}), 5.11\left(2 \mathrm{H}, \mathrm{s}, \mathrm{NH} \underline{\mathrm{CH}}_{2} \mathrm{Ph}\right), 3.37\left(4 \mathrm{H}, \mathrm{t}, 2 \mathrm{x}\left(\mathrm{CONH} \underline{C H}_{2}\right), J 7.2 \mathrm{~Hz}\right), 2.52(4 \mathrm{H}, \mathrm{t}$, $\left.2 \mathrm{x}\left(\underline{\mathrm{CH}}_{2} \mathrm{~N}\left(\mathrm{CH}_{3}\right)_{2}\right), J 7.2 \mathrm{~Hz}\right), 2.30\left(12 \mathrm{H}, \mathrm{s}, 4 \mathrm{xCH}_{3}\right) .{ }^{13} \mathrm{C} \mathrm{NMR}\left(150 \mathrm{MHz}, \mathrm{CD}_{3} \mathrm{OD}\right): \delta_{\mathrm{C}} 157.1$ (CO), 156.8 (C9), 147.1 (C3, C6), 143.4 (C4a, C10a), 138.6 (C1'), 130.2 (C3', C5'), 128.9 (C4'), 127.6 (C2', C6), 127.1 (C1, C8), 117.1 (C2, C7), 108.8 (C8a, C9a), 104.3 (C4, C5), 59.8 $\left(\underline{\mathrm{CH}}_{2} \mathrm{~N}\left(\mathrm{CH}_{3}\right)_{2}\right), 52.8\left(\mathrm{NH} \underline{C H}_{2} \mathrm{Ph}\right), 45.6\left(\mathrm{CH}_{3}\right), 38.5(\mathrm{CONH} \underline{\mathrm{CH}} 2)^{2}$. Anal. Calcd for $\mathrm{C}_{30} \mathrm{H}_{38} \mathrm{~N}_{8} \mathrm{O}_{2}$ (542.68): C, 66.40; H, 7.06; N, 20.65\%. Found: C, 66.27; H, 7.14; N, 20.36\%.

3,6-Bis\{3-[2-(dimethylamino)ethyl]ureido\}-9-[(2-phenylethyl)amino]acridine (18b). Yield 40\%, 0.20 g. ${ }^{1} \mathrm{H}$ NMR $\left(600 \mathrm{MHz}, \mathrm{CD}_{3} \mathrm{OD}\right): \delta_{\mathrm{H}} 7.77(2 \mathrm{H}, \mathrm{d}, \mathrm{H}-1,8, J 9.6 \mathrm{~Hz}), 7.65$ (2H, d, H$4,5, J 1.8 \mathrm{~Hz}), 7.20-7.10\left(7 \mathrm{H}, \mathrm{m}, \mathrm{H}-2,7, \mathrm{H}-2^{\prime}, 6, \mathrm{H}-3^{\prime}, 5^{\prime}, \mathrm{H}-4^{\prime}\right), 3.89$ (2H, t, $\mathrm{NH}^{\mathrm{CH}} \underline{2}_{2} \mathrm{CH}_{2} \mathrm{Ph}, J$ $7.2 \mathrm{~Hz}), 3.35\left(4 \mathrm{H}, \mathrm{t}, 2 \mathrm{x}\left(\mathrm{CONH} \underline{C H}_{2}\right), J 7.2 \mathrm{~Hz}\right), 2.98\left(2 \mathrm{H}, \mathrm{t}, \mathrm{NHCH}_{2} \underline{\mathrm{CH}}_{2} \mathrm{Ph}, J 7.2 \mathrm{~Hz}\right), 2.49(4 \mathrm{H}$, $\left.\mathrm{t}, 2 \mathrm{x}\left(\underline{\mathrm{CH}}_{2} \mathrm{~N}\left(\mathrm{CH}_{3}\right)_{2}\right), J 7.2 \mathrm{~Hz}\right), 2.26\left(12 \mathrm{H}, \mathrm{s}, 4 \mathrm{xCH}_{3}\right) .{ }^{13} \mathrm{C} \mathrm{NMR}\left(150 \mathrm{MHz}, \mathrm{CD}_{3} \mathrm{OD}\right): \delta_{\mathrm{C}} 157.4$ (CO), 154.5 (C9), 146.0 (C4a, C10a), 145.5 (C3, C6), 139.4 (C1'), 129.8 (C2', C6'), 129.7 (C3', C5'), 127.7 (C4), 126.2 (C1, C8), 117.2 (C2, C7), 110.1 (C8a, C9a), 107.3 (C4, C5), 59.8 $\left(\underline{\mathrm{CH}}_{2} \mathrm{~N}\left(\mathrm{CH}_{3}\right)_{2}\right), 51.5\left(\mathrm{NH} \underline{C H}_{2} \mathrm{CH}_{2} \mathrm{Ph}\right), 45.6\left(\mathrm{CH}_{3}\right), 38.6\left(\mathrm{CONH} \underline{C H}_{2}\right), 37.4\left(\mathrm{NHCH}_{2} \underline{\mathrm{CH}}_{2} \mathrm{Ph}\right)$. Anal. Calcd for $\mathrm{C}_{31} \mathrm{H}_{40} \mathrm{~N}_{8} \mathrm{O}_{2}$ (556.70): C, 66.88; H, 7.24; N, 20.13\%. Found: C, 66.73; H, 7.35; $\mathrm{N}, 19.87 \%$.

3,6-Bis $\{3-[2-($ dimethylamino)ethyl]ureido\}-9-[(3-phenylpropyl)amino]acridine (18c). Yield $33 \%, 0.18$ g. ${ }^{1} \mathrm{H}$ NMR $\left(600 \mathrm{MHz}, \mathrm{CD}_{3} \mathrm{OD}\right): \delta_{\mathrm{H}} 7.69-7.67(4 \mathrm{H}, \mathrm{m}, \mathrm{H}-1,8, \mathrm{H}-4,5), 7.19-7.17$ $\left(2 \mathrm{H}, \mathrm{m}, \mathrm{H}-3^{\prime}, 5^{\prime}\right), 7.11$ - $7.06(5 \mathrm{H}, \mathrm{m}, \mathrm{H}-2,7, \mathrm{H}-2,6, \mathrm{H}-4), 3.62-3.60$ (2H, m, $\left.\mathrm{NH}_{\underline{C H}}{ }_{2} \mathrm{CH}_{2} \mathrm{CH}_{2} \mathrm{Ph}\right), 3.36\left(4 \mathrm{H}, \mathrm{t}, 2 \mathrm{x}\left(\mathrm{CONH} \underline{C H}_{2}\right), J 7.2 \mathrm{~Hz}\right), 2.61\left(2 \mathrm{H}, \mathrm{t}, \mathrm{NHCH}_{2} \mathrm{CH}_{2} \underline{\mathrm{CH}_{2}} \underline{\mathrm{Ph}}_{2} J\right.$ $7.2 \mathrm{~Hz}), 2.49\left(4 \mathrm{H}, \mathrm{t}, 2 \mathrm{x}\left(\underline{\mathrm{CH}}_{2} \mathrm{~N}\left(\mathrm{CH}_{3}\right)_{2}\right), J 7.2 \mathrm{~Hz}\right), 2.26\left(12 \mathrm{H}, \mathrm{s}, 4 \mathrm{xCH}_{3}\right), 2.03-1.98(2 \mathrm{H}, \mathrm{m}$, $\left.\mathrm{NHCH}_{2} \underline{\mathrm{CH}_{2}} \mathrm{CH}_{2} \mathrm{Ph}\right) .{ }^{13} \mathrm{C} \mathrm{NMR}\left(150 \mathrm{MHz}, \mathrm{CD}_{3} \mathrm{OD}\right): \delta_{\mathrm{C}} 157.4$ (CO), 154.6 (C9), 145.9 (C4a, C10a), 145.5 (C3, C6), 142.1 (C1'), 129.5 (C2', C6', C3', C5'), 127.1 (C4), 126.2 (C1, C8), 117.0 (C2, C7), 109.8 (C8a, C9a), 107.1 (C4, C5), 59.8 (CONHCH$\left.\underline{C H}_{2}\right), 49.4\left(\mathrm{NH}_{\underline{C H}} \underline{\mathrm{CH}}_{2} \mathrm{CH}_{2} \mathrm{Ph}\right), 45.6$ $\left(\mathrm{CH}_{3}\right), 38.6\left(\underline{\mathrm{CH}}_{2} \mathrm{~N}\left(\mathrm{CH}_{3}\right)_{2}\right), 33.9\left(\mathrm{NHCH}_{2} \mathrm{CH}_{2} \mathrm{CH}_{2} \mathrm{Ph}\right), 32.7\left(\mathrm{NHCH}_{2} \underline{\mathrm{CH}}_{2} \mathrm{CH}_{2} \mathrm{Ph}\right)$. Anal. Calcd for $\mathrm{C}_{32} \mathrm{H}_{42} \mathrm{~N}_{8} \mathrm{O}_{2}$ (570.73): C, 67.34; H, 7.42; N, 19.63\%. Found: C, 67.53; H, 7.30; N, $19.50 \%$.

\section{Acknowledgements}

This study was supported by Slovak Research and Development Agency under contract VEGA grant No. 1/0672/11, and Internal Grant Program of the P. J. Šafárik University in Košice (VVGS-PF-2013-78). 


\section{References}

1. Belmont, P.; Bosson, J.; Godet, T.; Tiano, M. Anti-Cancer Agents Med. Chem. 2007, 7(2), 139-169.

http://dx.doi.org/10.2174/187152007780058669

2. Belmont, P.; Dorange, I. Expert Opin. Ther. Pat. 2008, 18(11), 1211-1224.

http://dx.doi.org/10.1517/13543776.18.11.1211

3. Gowan, S. M; Harrison, J. R.; Patterson, L.; Valenti, M.; Read, M. A.; Neidle. S.; Kelland, L. R. Mol. Pharmacol. 2002, 61, 1154-1160.

http://dx.doi.org/10.1124/mol.61.5.1154

4. Burger, A. M.; Dai, F.; Schultes, C. M.; Reszka, A. P.; Moore, M. J.; Double, J. A.; Neidle, S. Cancer Res. 2005, 65, 1489-1496.

http://dx.doi.org/10.1158/0008-5472

5. Gunaratnam, M.; Greciano, O.; Martins, C.; Reszka, A. P.; Schultes, C. M.; Morjani, H., Riou, J. F.; Neidle, S. Biochem. Pharmacol. 2007, 74, 679-689.

http://dx.doi.org/10.1016/j.bcp.2007.06.011

6. Leonetti, C.; Amodei, S.; D’Angelo, C.; Rizzo, A.; Benassi, B.; Antonelli, A.; Elli, R.; Stevens, M. F.; D'Incalci, M.; Zupi, G.; Biroccio, A. Mol. Pharmacol. 2004, 66, 1138-1146. http://dx.doi.org/10.1124/mol.104.001537

7. Gomez, D.; Wenner, T.; Brassart, B.; Douarre, C.; O’Donohue, M. F., El Khoury, V.; ShinYa, K.; Morjani, H.; Trentesaux, C.; Riou, J. F. J. Biol. Chem. 2006, 281, 38721-38729. http://dx.doi.org/10.1074/jbc.M605828200

8. Phatak, P.; Cookson, J. C.; Dai, F.; Smith, V.; Gartenhaus, R. B.; Stevens, M. F.; Burger, A. M. Br. J. Cancer 2007, 96, 1223-1233.

9. Mo, Y.; Gan, Y.; Song, S.; Johnston, J.; Xiao, X.; Wientjes, M. G. Cancer Res. 2003, 63, 579-585.

http://dx.doi.org/10.1158/0008-5472

10. De Cian, A.; Lacroix, L.; Douarre, C.; Temime-Smaali, N.; Trentesaux, C.; Riou, J. F.; Mergny, J. L. Biochimie 2008, 90, 131-155.

http://dx.doi.org/10.1016/j.biochi.2007.07.011

11. Oganesian, L.; Bryan, T. M. Bioessays 2007, 29, 155-615.

http://dx.doi.org/10.1002/bies.20523

12. Martinez-Mańez, R.; Sancenon, F. Chem. Rev. 2003, 103, 4419- 4476.

http://dx.doi.org/10.1021/cr010421e

13. Harrison, R. J.; Cuesta, J.; Chessari, G.; Read, M. A; Basra, S. K.; Reszka, A. P; Morrell, J.;

Gowan, S. M.; Incles, C. M.; Tanious, F. A; Wilson, W. D; Kelland, L. R.; Neidle, S. J. Med. Chem. 2003, 46, 4463-4476.

http://dx.doi.org/10.1021/jm0308693

14. Paul, A.; Ladame, S. Org. Lett. 2009, 11(21), 4894-4897.

http://dx.doi.org/10.1021/o19019925 
15. Šebestík, J.; Šafařík, M.; Stibor, I.; Hlaváček, J. Biopolymers 2006, 84, 605-614. http://dx.doi.org/10.1002/bip.20590

16. MOPAC2012, Stewart, J. J. P. Stewart Computational Chemistry, Version 13.159W.

17. Sattigeri, V. J.; Soni, A.; Singhal, S.; Khan, S.; Pandya, M.; Bhateja, P.; Mathur, T.; Rattan, A.; Khanna, J.; Mehta, A. Arkivoc 2005, 2, 46-59.

18. Ghosh, H.; Yella, R.; Nath, J.; Patel, B. K. Eur. J. Org. Chem. 2008, 6189-6196. http://dx.doi.org/10.1002/ejoc.200800901

19. Sun, N.; Li, B.; Shao, J.; Mo, W.; Hu, B.; Shen, Z.; Hu, X. Beilstein J. Org. Chem. 2012, 8, 61-70. http://dx.doi.org/10.3762/bjoc.8.6

20. Lang, X.; Li, L.; Chen, Y.; Sun, Q.; Wu, Q.; Liu, F.; Tan, C.; Liu, H.; Gao, C.; Jiang, Y. Bio. Med. Chem. 2013, 21(14), $4170-4177$. http://dx.doi.org/10.1016/j.bmc.2013.05.008

21. Hedge, R.; Thimmaiah, P.; Yerigeri, M. C.; Krishnegowda, G.; Thimmaiah, K. N.; Houghton, P. J. Eur. J. Med. Chem. 2004, 39(2), 161 - 178.

http://dx.doi.org/10.1016/j.ejmech.2003.12.001 